\title{
Erratum to: Factors associated with hand joint destruction in Chinese patients with rheumatoid arthritis
}

Lijuan Zhang ${ }^{1,2 \dagger}$, Jing Wang ${ }^{3 \dagger}$, Qiuxiang Zhang ${ }^{1,2}$, Ting Fu ${ }^{1,2}$, Rulan Yin ${ }^{1,2}$, Ze Wang ${ }^{3}$, Liren $\mathrm{Li}^{2}$, Xianhua $\mathrm{Wu}^{3^{*}}$ and Zhifeng $\mathrm{Gu}^{1 *}$

\section{Erratum}

After publication of the original article [1] it was brought to our attention that author Jing Wang was incorrectly included as Jing wang. The correct spelling of the name is included in the author list of this erratum and updated in the original article.

\begin{abstract}
Author details
'Department of Rheumatology, Affiliated Hospital of Nantong University, 20th Xisi Road, 226001 Nantong, People's Republic of China. ${ }^{2}$ School of Nursing, Nantong University, 19th Qixiu Road, 226001 Nantong, People's Republic of China. ${ }^{3}$ Department of Medical Image, Affiliated Hospital of Nantong University, 20th Xisi Road, 226001 Nantong, People's Republic of China.
\end{abstract}

Received: 23 May 2017 Accepted: 23 May 2017

Published online: 30 May 2017

\section{Reference}

1. Zhang $L$, et al. Factors associated with hand joint destruction in Chinese patients with rheumatoid arthritis. BMC Musculoskelet. Disord. 2017;18:211 doi:10.1186/s12891-017-1548-7.

\footnotetext{
*Correspondence: wxh637295@sina.com; guzf@ntu.edu.cn

${ }^{\dagger}$ Equal contributors

${ }^{3}$ Department of Medical Image, Affiliated Hospital of Nantong University, 20th Xisi Road, 226001 Nantong, People's Republic of China

'Department of Rheumatology, Affiliated Hospital of Nantong University, 20th Xisi Road, 226001 Nantong, People's Republic of China

Full list of author information is available at the end of the article
} 